\title{
Synthesis, Morphology and Application of Alkylaryl Hyperbranched Polyesters
}

\author{
Li-Ming TANG, ${ }^{\dagger}$ Teng QIU, Xin-Lin TuO, \\ Xiao-Long ZHANG, and De-Shan LiU \\ Department of Chemical Engineering, School of Materials Science and Engineering, Tsinghua University, Beijing 100084, China
}

(Received August 10, 2001; Accepted November 22, 2001)

\begin{abstract}
Melt condensation of dipropyl 5-(hydroxyethoxy) isophthalate (1) and 5-hydroxyethoxy isophthalic acid (2) gave two soluble alkylaryl hyperbranched polyesters (defined as P1 and P2, respectively). Longer reaction time gave P2 with higher intrinsic viscosity and lower solubility in various solvents. Comparing the thermal properties of P1 with that of P2, it was shown that $T_{\mathrm{g}}$ and $T_{\mathrm{d}}$ were mainly depended on end-groups rather than molecular weight. Solid P2 formed from the melting state was composed of fine particles as observed by scanning electron microscopy (SEM). Ionized P2 was used as polyanion to form a self-assembled film with polycationic poly(diallydimethylammonium chloride) (PDAC) by layer-by-layer dipping. The layer formed from ionized P2 was observed by atomic force microscopy (AFM). Hyperbranched macromolecules thus formed spherical particles with uniform dimension. Particle size was 100 $225 \mathrm{~nm}$ with average particle size of $140 \mathrm{~nm}$ in the self-assembled film. P2 was a more effective rheology modificator for processing polycarbonate than $\mathrm{P} 1$.
\end{abstract}

KEY WORDS Hyperbranched Polymer/ Synthesis / Morphology / Rheology Modifier /

Hyperbranched polymers have obtained increasing interest during the past decade because of unique physical and chemical properties. ${ }^{1,2}$ They are mainly prepared by melt polycondensation of $\mathrm{AB}_{2}$ monomers and characterized by a large number of end-groups. The low viscosity as melt and in solution, high solubility and high reactivity of end-groups make them very attractive for use as coatings, thermosets, rheology modifiers and so on. Due to the ease in the synthesis of $\mathrm{AB}_{2}$ monomers derived from 3,5-dihydroxy benzoic acid and 5-hydroxy isophathalic acid, allaromatic hyperbranched polyesters have been studied in much detail. ${ }^{3,4}$ However, high reaction temperatures for synthesis often yield insoluble products owning to the cross-linking reaction between the terminal groups. A study showed that polycondensation of 5-(2hydroxyethoxy)isophthalic acid could be conducted at relatively lower temperature to yield a soluble alkylaryl hyperbranched polyester. ${ }^{5}$ However, studies on alkylaryl hyperbranched polyesters are still few.

An important feature for hyperbranched polymers is highly branched architecture, which affects morphology both as solid and in solvent. A recent article studies the molecular organization of hyperbranched poly(chloroethyl vinyl ether-g-styrene) copolymers in thin film by means of atomic force microscopy (AFM). ${ }^{6}$ Due to intrinsic molecular architecture, a "doughnut-like" morphology was observed. Actually, these polymers should be regarded as comb-like grafting polymers rather than hyperbranched polymers.

${ }^{\dagger}$ To whom correspondence should be addressed.
To our knowledge, no study has been made on the morphology of hyperbranched polymer based on $\mathrm{AB}_{2}$ monomer.

In this study, two alkylaryl hyperbranched polyesters were prepared by polycondensation of dipropyl 5(hydroxyethoxy)isophthalate (1) and 5-hydroxyethoxyisophthalic acid (2) under melting condition. The morphology of solid poly(5-hydroxyethoxyisophthalic acid) (P2) was observed by scanning electron microscopy (SEM). Ionized P2 was fabricated into selfassembled film by the layer-by-layer adsorption technique $^{7,8}$ with poly(diallydimethylammonium chloride) (PDAC). The surface of the self-assembled film with P2 as the outermost layer was observed by atomic force microscopy (AFM). Finally, the utility of these polymers as rheology modifiers for processing polycarbonate (PC) was studied.

\section{EXPERIMENTAL}

Preparation of Dipropyl 5-(2-hydroxyethoxy) isophthalate (1)

A solution of $20 \mathrm{~g}(110 \mathrm{mmol})$ of 5-hydroxyisophthalic acid in $200 \mathrm{~mL}$ of absolute propanol was refluxed in the presence of $7.5 \mathrm{~mL}$ of concentrated sulfuric acid for $20 \mathrm{~h}$. Propanol was removed by rotary evaporation. The residue was poured into excess water. White solid product precipitated from the solution was collected, washed carefully with water and dried to yield $27.5 \mathrm{~g}$ dipropyl(5-hydroxy)isophthalate (in 94\% yield). Mp: $68-70^{\circ} \mathrm{C},{ }^{1} \mathrm{H}$ NMR (DMSO- $\left.d_{6}, \delta\right): 0.96$ 
$\left(6 \mathrm{H}, \mathrm{CH}_{2} \mathrm{CH}_{2} \mathrm{CH}_{3}\right), 1.75\left(4 \mathrm{H}, \mathrm{OCH}_{2} \mathrm{CH}_{2} \mathrm{CH}_{3}\right), 4.24$ $\left(4 \mathrm{H}, \mathrm{OCH}_{2} \mathrm{CH}_{2} \mathrm{CH}_{3}\right), 7.60(2 \mathrm{H}$, aromatic), $7.95(1 \mathrm{H}$, aromatic), $10.29(1 \mathrm{H}, \mathrm{OH})$.

A solution of $10 \mathrm{~g}(37.7 \mathrm{mmol})$ of dipropyl(5-hydroxy)isophthalate, $4 \mathrm{~mL}(58.6 \mathrm{mmol}) 2$-chloroethanol, $25 \mathrm{~g}$ anhydrous potassium carbonate and a catalytic amount of potassium iodide in $150 \mathrm{~mL}$ of propanol was reacted at $90^{\circ} \mathrm{C}$ for $72 \mathrm{~h}$. Propanol was removed by rotary evaporation. The residual mixture was poured into excess water and the product was precipitated out by adjusting the $\mathrm{pH}$ value to $1-2$. The crude product was recrystallized twice with methanol to obtain $3.9 \mathrm{~g}$ of (1) in $35.2 \%$ yield. Mp: $41-43{ }^{\circ} \mathrm{C} ;{ }^{1} \mathrm{H}$ NMR (DMSO- $d_{6}$, $\delta): 0.99\left(6 \mathrm{H}, \mathrm{CH}_{2} \mathrm{CH}_{2} \mathrm{CH}_{3}\right), 1.75\left(4 \mathrm{H}, \mathrm{OCH}_{2} \mathrm{CH}_{2}\right)$, $3.75\left(2 \mathrm{H}, \mathrm{CH}_{2} \mathrm{CH}_{2} \mathrm{OH}\right), 4.05\left(2 \mathrm{H}, \mathrm{CH}_{2} \mathrm{CH}_{2} \mathrm{OH}\right), 4.25$ $\left(4 \mathrm{H}, \mathrm{OCH}_{2} \mathrm{CH}_{2}\right), 4.9(1 \mathrm{H}, \mathrm{OH}), 7.65(2 \mathrm{H}$, aromatic), $8.05(1 \mathrm{H}$, aromatic).

\section{Preparation of 5-(2-hydroxyethoxy)isophthalic Acid (2)}

First, $3.0 \mathrm{~g}(10.6 \mathrm{mmol})$ of (1) were dissolved in $50 \mathrm{~mL}$ THF. Then $3 \mathrm{~g}(75 \mathrm{mmol})$ of $\mathrm{NaOH}$ dissolved in $80 \mathrm{~mL}$ water were added into the mixture and reacted under refluxing for $72 \mathrm{~h}$. After cooling to room temperature, $\mathrm{pH}$ was adjusted to $1-2$ by the addition of hydrochloric acid. After removal of THF by rotary evaporation and addition of excess water, the crude product was precipitated as white crystal. The precipitate was filtrated, washed and dried to give $2.1 \mathrm{~g}(2)$ in $88 \%$ yield. Mp: $201-203^{\circ} \mathrm{C} ;{ }^{1} \mathrm{H}$ NMR (DMSO- $d_{6}, \delta$ ): 3.75 $\left(2 \mathrm{H}, \mathrm{CH}_{2} \mathrm{CH}_{2} \mathrm{OH}\right), 4.05\left(2 \mathrm{H}, \mathrm{CH}_{2} \mathrm{CH}_{2} \mathrm{OH}\right), 4.85(1 \mathrm{H}$, $\mathrm{OH}), 7.6(2 \mathrm{H}$, aromatic $), 8.0(1 \mathrm{H}$, aromatic $), 13.0(2 \mathrm{H}$, $\mathrm{COOH})$.

\section{Polycondensation of Monomer (1) to P1}

Monomer (1) $(3.0 \mathrm{~g}, 10.6 \mathrm{mmol})$ and a catalytic amount of $\mathrm{Zn}(\mathrm{OAc})_{2}$ were placed in a reaction flask equipped with a stirrer, a nitrogen inlet and an outlet tube. The flask was evacuated, flushed with nitrogen twice to remove air and placed in an oil bath preheated to $175^{\circ} \mathrm{C}$ under a slow nitrogen stream for $1.5 \mathrm{~h}$. Vacuum was then applied for $2 \mathrm{~h}$ to remove any propanol. Temperature was increased to $190^{\circ} \mathrm{C}$. Vacuum was retained for an additional $1.5 \mathrm{~h}$. After cooling, a glasslike pale solid product was obtained $(2.2 \mathrm{~g}, 91 \%$ yield). ${ }^{1} \mathrm{H}$ NMR (DMSO- $d_{6}, \delta$ ): $0.99(\mathrm{~b}, 3 \mathrm{H}), 1.75(\mathrm{~b}, 2 \mathrm{H})$, $3.75(\mathrm{~b}, 2 \mathrm{H}), 4.05(\mathrm{~b}, 2 \mathrm{H}), 4.25(\mathrm{~b}, 2 \mathrm{H}), 7.8-8.0$ (b, aromatic).

\section{Polycondensation of Monomer (2) to P2}

Monomer (2) $(1.5 \mathrm{~g}, 6.6 \mathrm{mmol})$ and a catalytic amount of $\mathrm{Zn}(\mathrm{OAc})_{2}$ were placed in the reaction flask as above. The flask was evacuated, flushed with nitrogen twice to remove air and placed in an oil bath pre- heated to $225^{\circ} \mathrm{C}$ under a slow nitrogen stream for $1.5 \mathrm{~h}$. Vacuum was applied for $2 \mathrm{~h}$. After cooling, $1.4 \mathrm{~g}$ glasslike pale solid product $\mathrm{P} 2\left(90 \%\right.$ yield) was obtained. ${ }^{1} \mathrm{H}$ NMR (DMSO- $\left.d_{6}, \delta\right): 4.5$ (b, 2H), 4.8(b, 2H), 7.8-8.1 (b, aromatic), 9.8(b, 1H).

\section{Formation of Self-Assembed Layers}

The concentration of $\mathrm{P} 2$ and poly(diallydimethylammonium chloride) (PDAC, $\left.M_{\mathrm{w}}: 200000-350000\right)$ in Milli-Q water (resistance $>18 \mathrm{M} \Omega \mathrm{cm}$ ) were adjusted to $0.1 \mathrm{mmol} \mathrm{L}^{-1}$. The solution of $\mathrm{P} 2$ was adjusted to $\mathrm{pH}=6-7$ by $\mathrm{NaOH}$ to ionized the carboxylic acid end-groups. Quartz slide $(50 \mathrm{~mm} \times 14 \mathrm{~mm} \times 0.8 \mathrm{~mm})$ served as adsorption substrates was first sonicated in $98 \% \mathrm{H}_{2} \mathrm{SO}_{4} / 30 \% \mathrm{H}_{2} \mathrm{O}_{2}$ solution (piranha solution) for $1 \mathrm{~h}$ and washed with excess Milli-Q water for several times and then sonicated in $\mathrm{H}_{2} \mathrm{O} / \mathrm{H}_{2} \mathrm{O}_{2} / \mathrm{NH}_{4} \mathrm{OH}(5: 1: 1)$ solution for $1 \mathrm{~h}$ followed with thorough rinsing and dried with air stream. Self-assembled layers of ionized P2 and PDAC on quartz slide was formed by the layerby-layer adsorption technique ${ }^{7,8}$ by alternately dipping the treated quartz slide in PDAC and P2 solutions with each for $10 \mathrm{~min}$. After each dipping, the slide was washed with excess Milli-Q water for 2 min and dried with air stream.

\section{Measurement}

The morphology of the surface of solid P2 was observed directly by SEM. The surface of the selfassembled film on quartz slide was observed by Atomic Force Microscope Nanoscope IIIa (AFM, tapping Mode). Hyperbranched polymers were used as rheology modifiers in processing polycarbonate (PC). To make PC mixed well with hyperbranched polymer, a few drops of paraffin were added to the mixture before measurement. The melting index (MI) was measured by $\mu$ PXRZ-400B Melting-Flow-Speed measurement meter. According to GB/T 3632 standard, load and temperature were set to $1.2 \mathrm{~kg}$ and $300^{\circ} \mathrm{C}$, respectively during measurement.

\section{RESULTS AND DISCUSSION}

$\mathrm{AB}_{2}$ monomer 5-hydroxyethoxy isophthalic acid was prepared in the literature. ${ }^{5}$ However high pressure must be applied for ethoxylating the phenolic $\mathrm{OH}$ of 5-hydroxyisophthalic acid with ethylene oxide. In the present study, a different synthetic approach shown in Scheme 1 was adopted for preparation of monomer dipropyl 5-(2-hydroxyethoxy)isophthalate (1) and 5hydroxyethoxyisophthalic acid (2). First, dipropyl (5hydroxy)isophthalate was prepared by esterification of 5-hydroxyisophthalic acid by propanol for protecting 


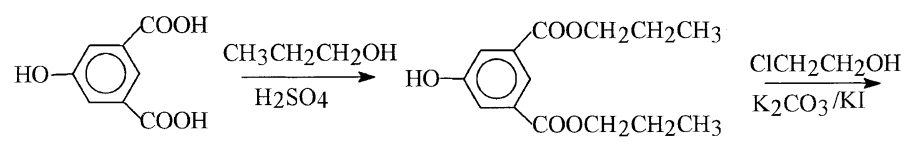

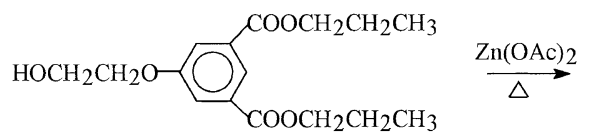

(1)

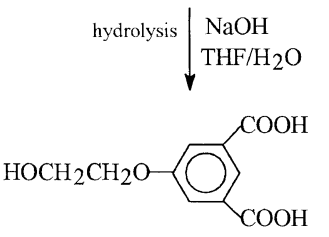

(2)

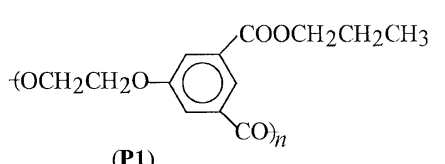

(P1)

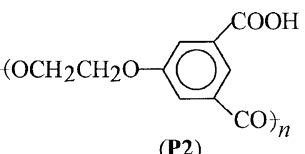

Scheme 1. Synthesis of the monomers and corresponding polymers.

Table I. Effects of reaction time under vacuum on the products properties

\begin{tabular}{ccccccc}
\hline No. & \multirow{2}{*}{ Time/h } & $\begin{array}{c}{[\eta]^{\mathrm{a}}} \\
\mathrm{dL} \mathrm{g}^{-1}\end{array}$ & $\begin{array}{c}M_{\mathrm{w}}^{\mathrm{b}} \\
/ 10^{4}\end{array}$ & $\begin{array}{c}\text { Solubility in } \\
\text { THF and DMF }\end{array}$ & $\begin{array}{c}\text { Solubility in } \\
\text { NaOH solution }\end{array}$ & $T_{\mathrm{d}}{ }^{\circ} \mathrm{C}$ \\
\hline 1 & 2 & 0.12 & 1.3 & slowly & easily & 414 \\
2 & 3 & 0.15 & 2.0 & slowly & normal & 417 \\
3 & 4 & 0.24 & 8.0 & very slowly & slowly & 430 \\
\hline
\end{tabular}

${ }^{a}$ Measured in DMF at $30.0^{\circ} \mathrm{C}$ at a concentration of $1.025 \mathrm{~g} \mathrm{~L}^{-1} .{ }^{\mathrm{b}}$ According to the relationship between $[\eta]$ and $M_{\mathrm{w}}$ developed by Turner. ${ }^{5}$

the two carboxylic acid groups. The reaction between dipropyl (5-hydroxy)isophthalate and 2-chloroethanol in propanol gave (1) in a yield of about $35 \%$. Further hydrolysis of (1) in basic THF/ $\mathrm{H}_{2} \mathrm{O}$ mixture yielded (2) in rather high yield (88\%). The structures of these products were confirmed by ${ }^{1} \mathrm{H}$ NMR.

Zinc acetate is a very effective catalyst for esterification, ${ }^{9}$ so it was adopted as catalyst for polycondensation of monomer (1) and (2). First, thermal polycondensation of monomer (1) was conducted at $190^{\circ} \mathrm{C}$ in the presence of $\mathrm{Zn}(\mathrm{OAc})_{2}$. The reaction initially took place under nitrogen atmosphere for $1.5 \mathrm{~h}$ and then under vacuum for $2 \mathrm{~h}$ to promote the reaction. The polymer (P1) could be dissolved completely in ethyl ether, DMF and THF. The intrinsic viscosity for P1 was $0.11 \mathrm{dL} \mathrm{g} \mathrm{g}^{-1}$ measured in DMF at a concentration of $1.025 \mathrm{~g} \mathrm{~L}^{-1}$. Thermal analysis of P1 showed $T_{\mathrm{g}}$ of $58^{\circ} \mathrm{C}$ and $T_{\mathrm{d}}$ of $365^{\circ} \mathrm{C}$. A $T_{\mathrm{g}}$ of $86^{\circ} \mathrm{C}$ for a similar polymer with methyl ester end-groups has been observed. ${ }^{10}$

Carboxylic acid terminated hyperbranched polyester (P2) was prepared from monomer (2) by relatively the same procedure as that for $\mathrm{P} 1$ at $225^{\circ} \mathrm{C}$ due to a high melting point $\left(201-203^{\circ} \mathrm{C}\right)$ of the monomer. Disappearance of the peak at around $5.0 \delta(\mathrm{OH})$ in the ${ }^{1} \mathrm{H}$ NMR spectrum indicates a rather high conversion in such system. By adjusting the reaction time under vacuum, three samples could be produced. Their properties were measured and summarized in Table I. Longer reaction time gave a product with higher intrinsic viscosity, i.e., higher molecular weight and consequently lower solubility in organic and alkali aqueous. Due to absorption of carboxylic acid terminated polymer to the separation column, molecular weight could not be analyzed directly by GPC. ${ }^{5}$ According to the relationship between $[\eta]$ and molecular weight established by Turner, ${ }^{5}$ the molecular weight of the present polymers could be estimated roughly as shown in Table I. The intrinsic viscosity of these polymers ranged from 0.12 to $0.24 \mathrm{dL} \mathrm{g} \mathrm{g}^{-1}$, indicating molecular weight to be higher than 10000 . Thermal analysis of these polymers showed $T_{\mathrm{g}}$ at around $176^{\circ} \mathrm{C}$ and $T_{\mathrm{d}}$ to slightly increase from $414^{\circ} \mathrm{C}$ to $430^{\circ} \mathrm{C}$ with respect to molecular weight shown in Table I. Comparing the results between P1 and $\mathrm{P} 2$, it is certain that the thermal properties of hyperbranched polyesters are mainly governed by the endgroups rather than the molecule weight, the same as found in other systems. ${ }^{10}$ For alkylaryl hyperbranched polyesters with spacer segment being incorporated, due to the difficulty in distinguishing the various types of segments by using NMR, the degree of branching could not be measured. ${ }^{11}$

With many terminal carboxylic acid groups, all the samples in Table I could be dissolved completely in $\mathrm{NaOH}$ aqueous solution at various dissolving rates. The number of terminal carboxylic acid groups could be measured by titration. ${ }^{12}$ To avoid hydrolysis of ester 


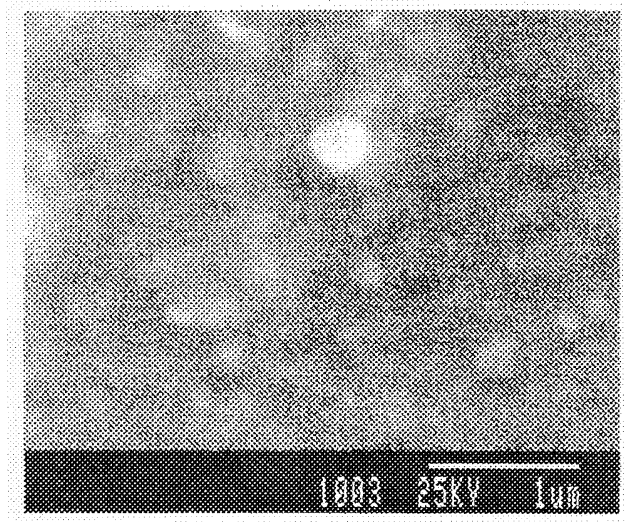

Figure 1. Morphology of solid P2 observed by SEM.

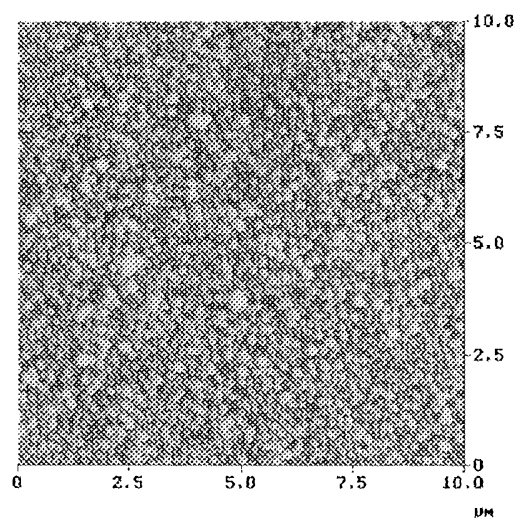

(a)

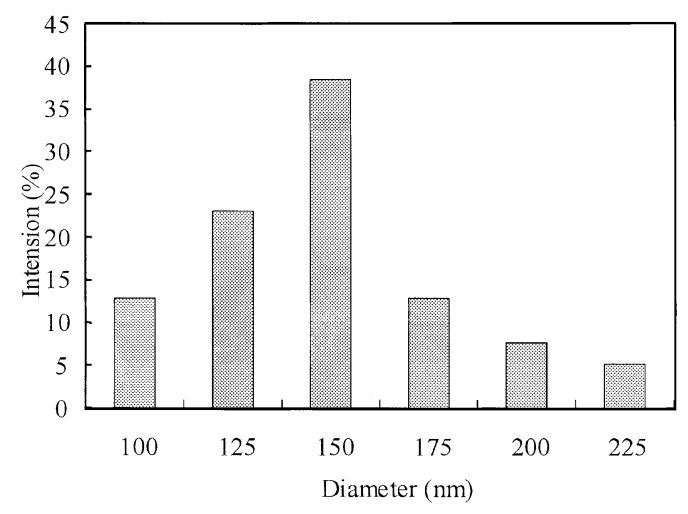

Figure 3. Size distribution of ionized $\mathrm{P} 2$ particles prepared by self-assembling.

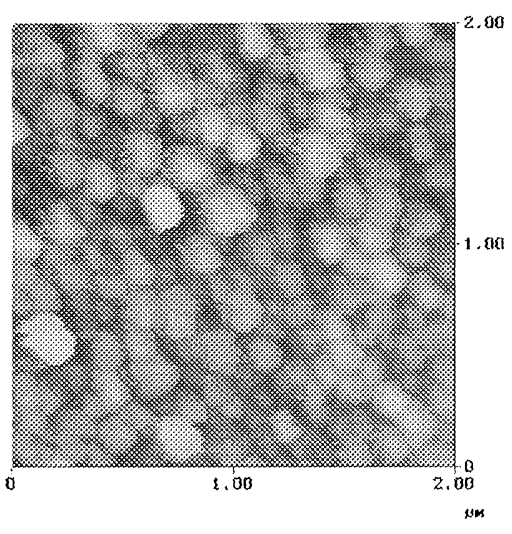

(b)

Figure 2. AFM morphology of the surface of ionized P2 formed by self-assembling.

groups during the measuring process, the sample with the highest dissolving rate was selected for measurement and thus a very short time was required for completing the titration. Based on the titration curve, ${ }^{12}$ terminal groups could be calculated to be $6.25 \mathrm{mmol} \mathrm{g}^{-1}$. If molecular weight could be measured, terminal carboxylic acid groups in each molecule could be calculated.

After cooling from the melting state, solid yellowish product was often formed which could be easily broken by hand. To study the morphology of hyperbranched polymer, a small piece of solid P2 was directly transferred to SEM observation. Figure 1 shows that hyperbranched polymer is composed of fine particles. However particle size could not be determined due to unclear morphology from the uneven surface. Direct observation by SEM thus may not be very suitable for solid hyperbranched polymers.

Self-assembling is well known for fabricating multilayers with perfect supramolecule structures. ${ }^{7,8}$ With plenty of terminal carboxylic acid groups, P2 could be transferred to the ionized form in aqueous $\mathrm{NaOH}$ solution. Because of opposite by charged attractive force between polymers, alternately dipping the sub- strate into dilute solutions of polyanions and polycations, uniform multilayers were formed from anionic P2 and cationic PDAC on a charged substrate slide. ${ }^{13}$ Characterization of assembled multilayers between P2 and PDAC has been reported elsewhere. ${ }^{12}$ Here the surface of PDAC and that of the ionized P2 formed by this process were observed by AFM to study morphology. The surface of $\mathrm{P} 2$ shown in Figure 2 was entirely composed of tiny spherical particles with rather uniform dimensions. Figure 3 shows particle size distribution of 100 to $225 \mathrm{~nm}$ and average particle size of $140 \mathrm{~nm}$. The surface of PDAC looks the same as that of P2 since it simply covers the surface of $\mathrm{P} 2$ as a very thin layer. Self-assembling technique is thus a feasible method for investigating the morphology of hyperbranched polymers.

Hyperbranched molecules may form spherical particles spontaneously as solid and in thin film irrelevant of charge density of terminal groups. The formation mechanism is still not clear. It is certain that morphology is affected by the structure of a hyperbranched polymer, such as the degree of branching and charging density of end-groups.

One possible application for hyperbranched poly- 


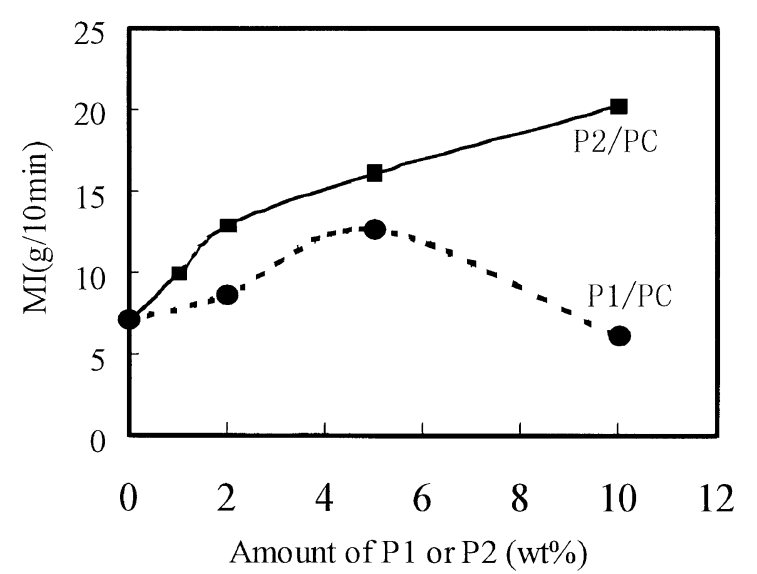

Figure 4. $\mathrm{MI}$ of $\mathrm{P} 1 / \mathrm{PC}$ and $\mathrm{P} 2 / \mathrm{PC}$ mixture.

mers is rheology modifier for thermoplastics. This use in linear thermoplastic polycarbonate (PC) was studied. The melting index (MI) for P2/PC and P1/PC systems is shown in Figure 4. With increasing $\mathrm{P} 2$ in the mixture, $\mathrm{MI}$ increased, indicating reduction in melt viscosity. At $\mathrm{P} 2$ of $5 \mathrm{wt} \%$ based on weight of PC, MI was 2.27 times larger than that of pure PC. The influence of $\mathrm{P} 2$ on color and transparence of the extruder was not observed in any study. P2 is thus a very effective rheology modifier in processing of $\mathrm{PC}$. For $\mathrm{P} 1 / \mathrm{PC}$, increase in MI was not so remarkable as in P2. This might be related to non-polar terminal groups in $\mathrm{P} 1$, with consequent poor miscibility with PC.

\section{REFERENCES}

1. B. Voit, J. Polym. Sci., Part A: Polym. Chem., 38, 2505 (2000).

2. K. Inoue, Prog. Polym. Sci., 25, 453 (2000).

3. A. Mock, A. Burgath, R. Hanselmann, and H. Frey., Macromolecules, 34, 7692 (2001).

4. H. R. Kricheldorf and O. Stober, Macromol. Rapid Commun., 15, 87 (1994).

5. S. R. Turner, F. Walter, B. I. Voit, and T. H. Mourey, Macromolecules, 27, 1611 (1994).

6. P. Viville, A. Deffieux, M. Schappacher, J. L. Bredas, and R. Lazzaroni, Mat. Sci. Eng., C, 15, 311 (2001).

7. G. Decher, J. D. Hong, and J. Schmitt, Thin Solid Films, 210/211, 831 (1992).

8. G. Decher, Science, 277, 1232 (1997).

9. K. Tomita and H. Ida, Polymer, 16, 185 (1975).

10. D. Parker and W. J. Feast., Macromolecules, 34, 2048 (2001).

11. A. Kumar and S. Ramakrishnan, Macromolecules, 29, 2534 (1996).

12. T. Qiu, L. M. Tang, X. L. Tuo, X. L. Zhang, and D. S. Liu, Polym. Bull., 47, 337 (2001).

13. L. F. Wu, X. L. Tuo, H. Cheng, X. G. Wang, and D. S. Liu, Macromolecules, 34, 8005 (2001). 